\title{
Modalités de formation des veines de quartz aurifère du district d'El Callao (Venezuela) : études texturales et des inclusions fluides
}

\author{
Aurélie Blanchet*, Alain Chauvet \\ FRE 2124, ISTO, université d'Orléans, 45060 Orléans cedex 2, France
}

Reçu le 31 janvier 2000 ; accepté le 17 avril 2000

Présenté par Jean Aubouin

\begin{abstract}
The El Callao district (Venezuela) gold-bearing quartz veins mode of formation: textural and fluid inclusion studies. Gold-bearing quartz veins from the El Callao district (Guyana Shield, Venezuela) are filled with several comb quartz generations. Their formation results from successive opening and filling stages during which low salinity mineralized fluids composed by $\mathrm{CO}_{2}-\mathrm{H}_{2} \mathrm{O}-\mathrm{salt}-\left( \pm \mathrm{CH}_{4}\right)$ were trapped. (c) 2000 Académie des sciences / Éditions scientifiques et médicales Elsevier SAS
\end{abstract}

gold / quartz veins / texture / fluid inclusions / Venezuela

Résumé - Les veines de quartz aurifères du district d'El Callao (bouclier Guyanais, Venezuela) se composent essentiellement de quartz en peigne, plus ou moins recristallisés. Plusieurs générations ont été définies, démontrant ainsi que leur formation résulte d'ouvertures successives. L'étude des inclusions fluides associées à ces différentes générations de quartz montre que des fluides à $\mathrm{CO}_{2}-\mathrm{H}_{2} \mathrm{O}-$ sels- $-\left( \pm \mathrm{CH}_{4}\right)$, de faible salinité, accompagnent la formation de ces veines. (C) 2000 Académie des sciences/Éditions scientifiques et médicales Elsevier SAS

or / veines de quartz / texture / inclusions fluides / Venezuela

\section{Abridged version}

\section{Introduction}

Structural study and detailed microtectonic analyses are important and necessary steps in the understanding of gold-bearing quartz vein formation. The association with fluid inclusions analysis allows us to estimate the physico-chemical conditions that prevailed during vein formation and consequently, to retrace the complete evolution of the deposit.

\section{Geological setting}

The Lo Increible district is composed of granitogneissic basement (Supamo complex, figure 1a), sur- rounded by sedimentary (El Callao formation) and volcano-sedimentary units (Cicapra and Yuruari formations) (figure 1b). Both are affected by a greenschist facies metamorphism [2]. Foliation is oriented east-west. The Lo Increible deposits are localized along the thrust contact that separates the Yuruari meta-sediments from the El Callao meta-andesites (figure 1c).

\section{Quartz veins}

Mineralizations occur under the form of quartz veins localized along tectonic contacts (figure 2a) or thick boudins parallel to the foliation (figure 2b). Vein mineralogy is poor. Quartz is dominant and carbonate and sulphides are minor. Gold is probably disseminated in quartz grains although native gold grains have been found in fractures that affect a pyrite grain.

\footnotetext{
* Correspondance et tirés à part : blanchet@geol.u-psud.fr

Adresse actuelle: UMR 8616, Laboratoire de géochimie des roches sédimentaires, bât. 504, université Paris-Sud, 91405 Orsay, France
} 


\subsection{Textural study}

Comb quartz grains filled the analysed veins (figure 2c). They become larger in size towards the core of the veins. Because comb quartz characterizes syn-opening crystallisation [5], the two different directions of quartz elongation are assimilated to opening directions. These two opening stages are respectively marked by a first comb quartz generation (Q1) and secondary comb quartz (Q2, figure 2c) or quartz overgrowths (figure 2d).

\subsection{Fluid inclusions study}

Fluid inclusions of samples from La Loca and Mina A mines have been analysed by microthermometric and Raman methods (table). Salinities and densities have been calculated with the MacFlincor and Clatra softwares [4, 6] using the equations of Borisenko [3] and Kerrick and Jacobs [8]. Based on their appearance at room temperature, three families of inclusions have been distinguished. Family I, the most abundant, are biphase $\mathrm{CO}_{2}-\mathrm{H}_{2} \mathrm{O}-$ salts- $\left( \pm \mathrm{CH}_{4}\right)$ inclusions. They occur as clusters in comb quartz Q1 and Q2 and along intragranular cracks within quartz overgrowths. These inclusions display a variable $\mathrm{CO}_{2}-\mathrm{H}_{2} \mathrm{O}$ ratio. Family II inclusions are monophase and filled by $\mathrm{CO}_{2}-\left( \pm \mathrm{CH}_{4}\right)$ fluids. They occur under similar habits of the family I ones. Family III inclusions are biphase and contain $\mathrm{H}_{2} \mathrm{O}$-salts- $\left( \pm \mathrm{CH}_{4}\right)$ with very poor volatile phase. They occur along intergranular cracks within all kinds of quartz. For the three families of inclusions, those from the La Loca mine contain significant amount of $\mathrm{CH}_{4}$ (table).

\subsection{Relationships between fluid inclusions and quartz texture}

Family I and II inclusions both occur as clusters in comb quartz Q1 and Q2 (figure 2e). They are systematically elongated perpendicular to the long axes of quartz grains, i.e. perpendicular to the growth direction (figure 3). Inversely, within quartz overgrowths, family I and II inclusions were found along intragranular trails limited in extension to the limits of the hosted grain (figure 2f). According to Goldstein and Reynolds [7], Roedder [10] and $\mathrm{Xu}$ [11], family I and II inclusions could be referred to as primary ones when occurring elongated within quartz grains and as pseudosecondary ones in the case of overgrowths. In both cases, the fluids could be assumed to be contemporaneous of quartz crystallization. Because family III inclusions always occur as trails that crosscut the grains boundaries (figure $2 \mathrm{~g}$ ) they are interpreted as secondary ones, entrapped posterior to the vein formation.

\section{Vein formation model and discussion}

Two main stages of opening and quartz crystallization have been determined by the quartz texture analysis developed in this work (figure 4). During the first one, earlier comb quartz (Q1) crystallizes with coeval trapping of $\mathrm{CO}_{2}-\mathrm{H}_{2} \mathrm{O}-$ salts- $\left( \pm \mathrm{CH}_{4}\right)$ fluids (families I and II inclusions). The second stage is marked by the crystallization of an additional comb quartz (Q2) or by quartz overgrowth development (figure 4). Similar fluids (family I and II inclusions) as during stage I were captured. Finally, the trapping of $\mathrm{H}_{2} \mathrm{O}-$ salts- $\left( \pm \mathrm{CH}_{4}\right)$ fluids (family III) occurred during the formation of late microfractures.

Our results demonstrate that family I and II fluid inclusions both occur under the same habit during stage 1 (elongated clusters) and stage 2 (intragranular planes) of the vein formation. The co-existence of two different fluid inclusion families could result from either heterogeneous trapping of two parent fluids, post-entrapment modifications (leakage and necking-down, [10]) or unmixing of an initially homogeneous fluid in two immiscible phases (see review in [1]). Because criteria described by Ramboz et al. [9] for fluid immiscibility and evidence for post-entrapment modifications have not been encountered, heterogeneous trapping of the two different fluids appears the most probable hypothesis. We conclude that polyphase opening and crystallization stages during which $\mathrm{CO}_{2}-\mathrm{H}_{2} \mathrm{O}-$ salts- $\left( \pm \mathrm{CH}_{4}\right)$ fluids were captured formed gold-bearing quartz veins of the El Callao district. This example allows a good estimation of the evolution of fluid and physico-chemical conditions during the formation of quartz mineralized veins and perfectly illustrates the interest of combining textural and fluid inclusions studies.

\section{Introduction}

L'étude structurale et géométrique des veines de quartz aurifères est à la base de la compréhension des processus de formation des minéralisations filoniennes. L'analyse microtectonique détaillée (état de déformation, orientation des grains, relations avec l'orientation préférentielle des axes $\langle c\rangle$ ) apporte des contraintes pour l'établissement d'un modèle d'ouverture des veines. L'association de cette analyse à l'étude des inclusions fluides permet d'estimer les conditions physico-chimiques contempo- raines de la formation des veines. Ce travail présente un exemple d'analyse combinée de la texture des grains de quartz et des inclusions fluides réalisées sur les filons de quartz aurifères du district d'El Callao (bouclier paléoprotérozoïque Guyanais, Venezuela).

\section{Cadre géologique}

Le district aurifère $d^{\prime} E l$ Callao renferme d'abondantes minéralisations qui ont produit à ce jour $180 \mathrm{t}$ d'or. La région est composée d'un socle granito-gneissique (com- 


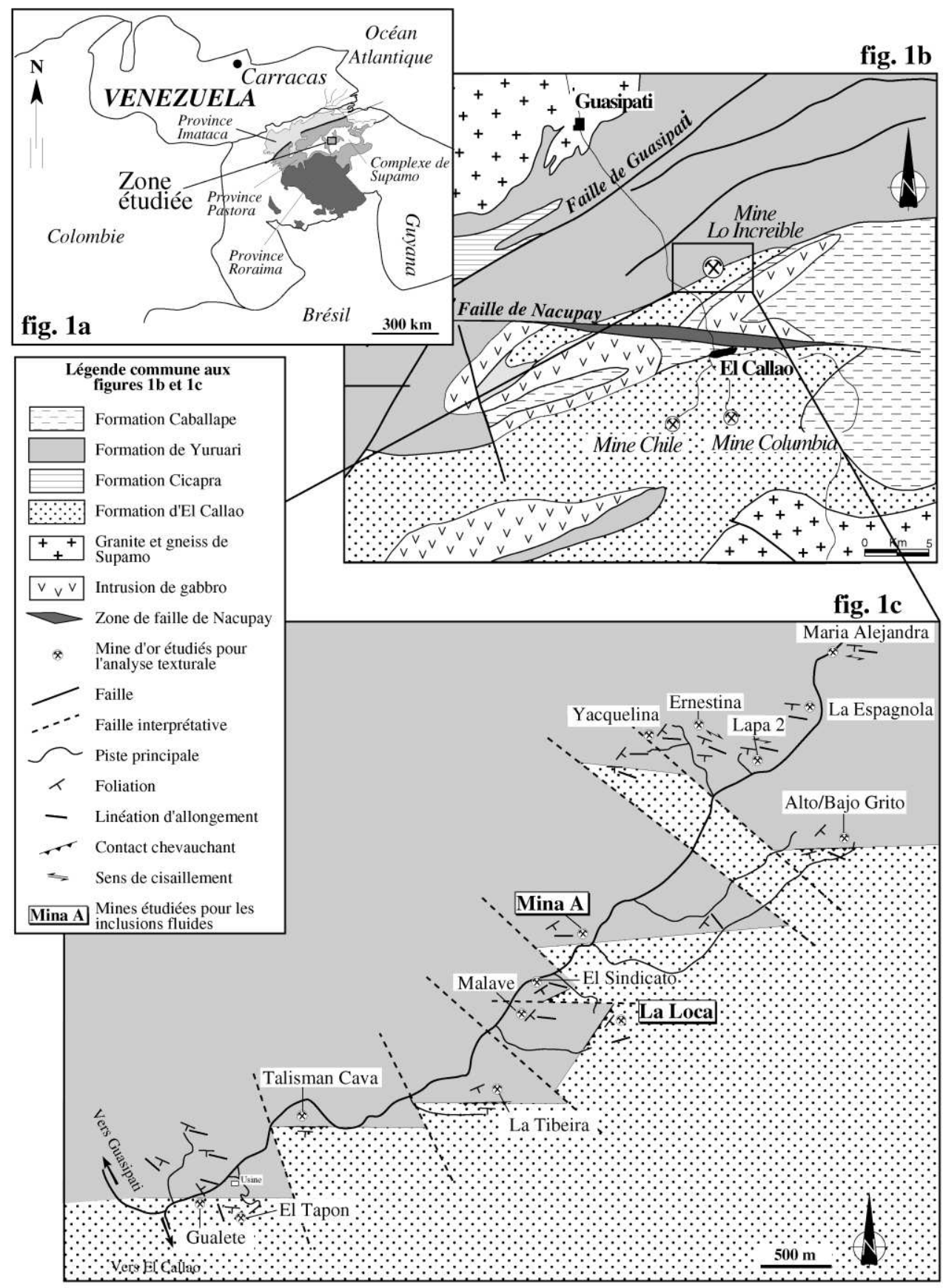

Figure 1. a. Carte des grandes provinces géologiques du Venezuela central. b. Carte géologique simplifiée du district d'El Callao. c. Carte géologique interprétative et schématique du district de Lo Increible. La localisation de tous les indices minéralisés utilisés pour l'étude texturale et des deux filons analysés pour les inclusions fluides est indiquée.

Figure 1. a. Map of the main geological provinces of central Venezuela. b. Geological map of the El Callao district. c. Interpretative and schematic geological map of the Lo Increible district. Location of analysed veins for textural and fluid inclusions studies is indicated. plexe de Supamo, figure 1a) et d'unités sédimentaires (formation El Callao) et volcano-sédimentaires (formations de Cicapra et Yuruari), datées entre 2,15 et 2,35 Ga [2] (figure 1b). L'ensemble de ces séries a subi un métamorphisme de faciès schistes verts, associé à une déformation responsable de la foliation orientée est-ouest à NNE-SSW. Les gisements étudiés sont localisés au contact entre les méta-sédiments de la formation de Yuruari et les méta-andésites de la formation d'El Callao (figures $1 b$ et $1 c)$. Ce contact montre des critères de chevauchement à vergence vers le nord-ouest (figure 2a). Le peu d'affleurements ne nous permettra pas de présenter une cartographie détaillée de la zone étudiée. Toutefois, il semblerait que l'ensemble du secteur soit affecté par un réseau de failles NW-SE, qui décalent l'interface sédiment-andésite et qui sont représentées de manière schématique sur la figure $1 c$.

\section{Les veines de quartz}

Les corps minéralisés se présentent sous la forme de veines d'épaisseur moyenne (de quelques centimètres à $1 \mathrm{~m}$ ) et de longueur décamétrique, parallèles ou sécan 

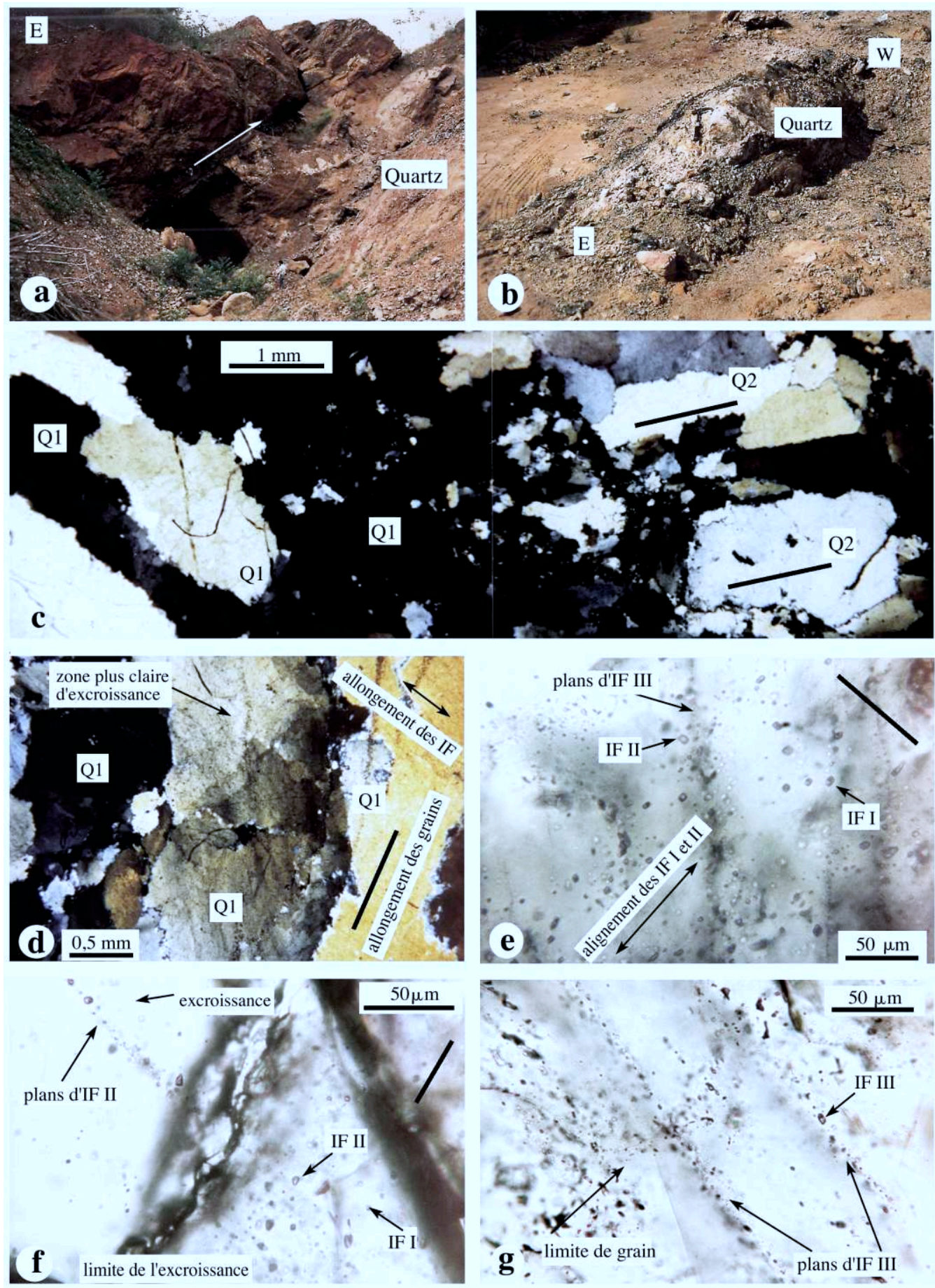

Figure 2. a. Filon de quartz dans le contact chevauchant de la mine La Loca. b. Boudin de quartz intra-foliation de la mine Maria Alejandra. c. Quartz en peigne montrant deux directions d'ouverture distinctes (secteur Ernestina). d. Quartz en peigne Q1 et zone d'excroissance (secteur La Loca). e. Inclusions fluides (IF) I et II allongées dans un grain Q1 et plan d'inclusions III (secteur Mina A). La ligne noire matérialise la direction de I'allongement du quartz Q1. f. IF II en amas dans un quartz Q1 et en plan au sein de l'excroissance (secteur La Loca). La ligne noire matérialise la direction de I'allongement du quartz Q1. g. IF III en plans intergranulaires (secteur La Loca).

Figure 2. a. Quartz vein located within thrust plane, La Loca mine. b. Intra-foliation quartz boudin from the Maria Alajandra mine. c. Two generations of comb quartz (Q1 and Q2) indicator of two directions of opening (Ernestina area). d. Comb quartz Q1 and quartz overgrowth (La Loca area). e. Family I and II fluid inclusions occurring within a Q1 comb quartz (Mina A area). Note the elongation of the inclusions I and II and the occurrence of a plane of family III inclusions. The black line materializes Q1 quartz grain elongation. f. Family II inclusions disseminated within quartz Q1 and along intragranular plane within quartz overgrowth (La Loca area). The black line materializes Q1 quartz grain elongation. g. Family III fluid inclusions within intergranular trails (La Loca area). 
tes sur la foliation. Ils sont fréquemment localisés le long des contacts tectoniques ou lithologiques (figure 2a), ou bien en boudins au cœur de la foliation (figure $2 b$ ). L'assemblage minéralogique est pauvre. Les veines sont principalement constituées de quartz et contiennent rarement des carbonates et de la pyrite altérée. II est probable que l'or soit disséminé dans les grains de quartz, bien que des plages d'or natif aient été trouvées dans les fractures d'une des rares pyrites observées.

\section{1. Étude texturale}

Les veines sont majoritairement constituées de quartz en peigne (figure 2c) (e.g. [5]). Les grains sont de forme allongée et leur taille, variable, augmente vers le cœur des veines. Les grains sont affectés par une déformation qui se traduit par une extinction roulante et une recristallisation devenant importante en bordure de veine. Les quartz en peigne sont caractéristiques d'une cristallisation contemporaine de l'ouverture ; la mesure de l'allongement des grains permet de matérialiser les directions d'ouverture. Dans certains échantillons, deux générations de quartz en peigne d'orientations différentes ont été reconnues (Q1 et Q2), indiquant ainsi l'existence de deux stades d'ouverture (figure 2c). Dans d'autres cas, le fait que la présence d'excroissances de quartz sur les grains Q1 ne soit pas en continuité optique avec le grain parent traduit les effets de la deuxième phase d'ouverture et de cristallisation (figure $2 d$ ). Ces excroissances ne se rencontrent qu'au cœur des veines. Ce deuxième événement se traduit, en bordure des veines, par une seconde cristallisation de quartz en peigne Q2 (figure 4).

\section{2. Étude des inclusions fluides}

Sur la base de l'analyse texturale, les échantillons de deux mines, la Mina A (inclusions indexées a) et La Loca (inclusions indexées b), ont été sélectionnés pour l'étude des inclusions fluides (voir localisation sur la figure 1c). Le filon de la Loca, d'épaisseur métrique, est localisé sur un contact chevauchant (figure 2a). Les veines de quartz de la Mina $A$ sont de taille centimétrique et sont localisées dans les andésites situées au-dessus du contact avec les méta-sédiments. Les mesures microthermométriques ont été effectuées sur des sections polies double-face, d'environ $200 \mu \mathrm{m}$ d'épaisseur, à l'aide d'une platine Chaixmeca. Le logiciel MacFlincor [4] a été utilisé pour le calcul des densités et des salinités.

\subsection{1. Étude pétrographique et microthermométrique}

À partir de l'observation microscopique et des résultats microthermométriques résumés dans le tableau, trois familles d'inclusions fluides ont été distinguées.

- Famille I. Ces inclusions, biphasées à $\mathrm{CO}_{2}-\mathrm{H}_{2} \mathrm{O}$-sels- $\left( \pm \mathrm{CH}_{4}\right)$, sont les plus abondantes. De taille moyenne ( 4 à $8 \mu \mathrm{m}$ ) et de forme tabulaire ou amiboïdale, elles sont, soit dispersées, soit disposées le long de microfractures intragranulaires (figure 2e) et montrent de grandes variations de rapport volumique vapeur/ liquide $(V / L$ de 0,1 à 0,7$)$. La température de première fusion de la glace, difficile à observer, a été mesurée entre -55 et $-52{ }^{\circ} \mathrm{C}$. Ces températures d'eutectique $\left(T_{\mathrm{e}}\right)$ suggèrent que nous sommes en présence $d^{\prime}$ une solution complexe, dont la composition, d'après les données de Borisenko [3], peut être à $\mathrm{H}_{2} \mathrm{O}+\mathrm{CaCl}_{2}+\mathrm{NaCl}$ $\left(T_{\mathrm{e}}=-55^{\circ} \mathrm{C}\right)$. Les salinités ont été calculées, pour les inclusions de la Mina A (la), grâce aux équations de Kerrick et Jacobs [8] appliquées aux températures de fusion du clathrate et, pour les inclusions de La Loca (Ib), avec le programme Clatra, qui tient compte de la présence de $\mathrm{CH}_{4}$ [6]. Des valeurs moyennes de 4,2 et $1,3 \%$ eq. poids $\mathrm{NaCl}$ ont été obtenues respectivement (tableau). Les données microthermométriques de la phase volatile divergent pour les deux mines. À La Loca (inclusions $\mathrm{Ib})$, les températures de fusion du $\mathrm{CO}_{2}\left(T_{\mathrm{f}_{\mathrm{CO}}}\right)$ sont comprises entre -68 et $-60{ }^{\circ} \mathrm{C}$, traduisant la présence de méthane dans la phase volatile, ce qui a été confirmé par I'analyse Raman (tableau). La moyenne des températures d'homogénéisation du $\mathrm{CO}_{2}$ est de $16^{\circ} \mathrm{C}$ et I'homogénéisation totale des inclusions $\mathrm{lb}$ se réalise entre 260 et $370{ }^{\circ} \mathrm{C}$ (tableau). Pour les échantillons de la Mina $\mathrm{A}$, la phase volatile est à $\mathrm{CO}_{2}$ pur, comme le montrent les valeurs de $T_{\mathrm{f}_{\mathrm{CO}_{2}}}$, proches de $-56,6^{\circ} \mathrm{C}$. La température d'homogénéisation du $\mathrm{CO}_{2} \mathrm{n}^{\prime}$ a pas pu être mesurée à cause de la très faible teneur en $\mathrm{CO}_{2}$ liquide. Ces inclusions fluides homogénéisent en phase liquide, à des températures qui s'étalent de $175^{\circ} \mathrm{C}$ à $325^{\circ} \mathrm{C}$ (tableau).

- Famille II. Les inclusions de cette famille sont monophasées, de taille plus petite $(<4 \mu \mathrm{m})$ et de forme ovö̈de, hexagonale ou carrée. Leur habitus est identique aux inclusions de la famille I (figures $2 e$ et $f$ ). Les inclusions de la Mina A (famille Ila) sont à $\mathrm{CO}_{2}$ pur, alors que celles de la Loca (famille IIb) contiennent du $\mathrm{CH}_{4}$ $\left(-66,5<T_{\mathrm{f}_{\mathrm{CO}_{2}}}<-61,5^{\circ} \mathrm{C}\right) \quad$ (tableau). La température $\mathrm{d}^{\prime}$ homogénéisation du $\mathrm{CO}_{2}$ se situe vers $-4,6^{\circ} \mathrm{C}$ pour les inclusions Ila. Une densité du $\mathrm{CO}_{2}$ de 0,95 est alors calculée [8]. Les données Raman montrent que les inclusions $\mathrm{Ilb}$ contiennent du $\mathrm{CH}_{4}$ et du $\mathrm{CO}_{2}$. Leur homogénéisation s'effectue, soit en phase liquide vers $-23^{\circ} \mathrm{C}$, soit en phase vapeur à $-16^{\circ} \mathrm{C}$; une densité totale moyenne de 0,52 a été calculée. La température de fusion du $\mathrm{CO}_{2}$ étant égale à $-56,6{ }^{\circ} \mathrm{C}$ pour les inclusions Ila, leur analyse Raman n'a pas été réalisée.

- Famille III. Les inclusions de la troisième famille sont de petite taille $(<4 \mu \mathrm{m})$ et de forme ovoïde ou fuselée. Ces inclusions biphasées composées de $\mathrm{H}_{2} \mathrm{O}-$ sels- $\left( \pm \mathrm{CH}_{4}\right)$ et à faible phase volatile sont systématiquement disposées dans des plans recoupant les limites de grain des quartz (figures $2 e$ et $2 g$ ). La faible dépression du point de fusion de la glace $\left(T_{\mathrm{m} 2}\right)$, mesurée à la fois pour les inclusions de la Mina A et pour celles de La Loca $\left(-2,5<T_{\mathrm{m} 2}<0\right)$ indique une très faible salinité (moyenne de $1,8 \%$ eq. $\mathrm{NaCl}$ ) (tableau). La présence de $\mathrm{CH}_{4}$ a été détectée en spectrométrie Raman uniquement dans les inclusions IIIb de la mine La Loca. 
A. Blanchet, A. Chauvet / C. R. Acad. Sci. Paris, Sciences de la Terre et des planètes / Earth and Planetary Sciences 330 (2000) $675-682$

Tableau. Typologie et composition des inclusions fluides (analyses microthermométriques et Raman). Les moyennes sont indiquées entre parenthèses.

Table. Fluid inclusion composition and typology (microthermometric and Raman analyses). Average values are indicated in brackets.

\begin{tabular}{|c|c|c|c|c|c|c|}
\hline & \multicolumn{6}{|c|}{ Typologie } \\
\hline & $I a$ & $I b$ & IIa & $I I b$ & III $a$ & $I I I b$ \\
\hline & Mina A & La Loca & Mina A & La Loca & Mina A & La Loca \\
\hline Aspect & $\theta \theta$ & $\theta \theta$ & 00 & 00 & $\circ 0$ & $\circ 0$ \\
\hline Composition & $\begin{array}{c}\mathrm{H}_{2} \mathrm{O}-\mathrm{CO}_{2} \\
\mathrm{CaCl}_{2}-\mathrm{NaCl} \\
\end{array}$ & $\begin{array}{c}\mathrm{H}_{2} \mathrm{O}-\mathrm{CO}_{2}-\mathrm{CaCl}_{2} \\
\mathrm{NaCl}-\mathrm{CH}_{4}\end{array}$ & $\mathrm{CO}_{2}$ & $\mathrm{CO}_{2}-\mathrm{CH}_{4}$ & $\mathrm{H}_{2} \mathrm{O}$-sels & $\mathrm{H}_{2} \mathrm{O}$-sels- $\mathrm{CH}_{4}$ \\
\hline Phases & \multicolumn{2}{|c|}{ biphasée } & \multicolumn{2}{|c|}{ monophasée } & \multicolumn{2}{|c|}{ biphasée } \\
\hline Forme & \multicolumn{2}{|c|}{ tabulaire, amiboïdale } & \multicolumn{2}{|c|}{$\begin{array}{c}\text { carrée, hexagonale ou } \\
\text { ovoüde }\end{array}$} & \multicolumn{2}{|c|}{ ovoüde, fuselée } \\
\hline Taille & \multicolumn{2}{|c|}{$4 \grave{a} 8 \mu \mathrm{m}$} & \multicolumn{2}{|c|}{$<4 \mu m$} & \multicolumn{2}{|c|}{$<4 \mu m$} \\
\hline Habitus & \multicolumn{2}{|c|}{$\begin{array}{c}\text { isolé, en amas ou en plans } \\
\text { intragranulaires }\end{array}$} & \multicolumn{2}{|c|}{$\begin{array}{c}\text { isolé ou en plans intra- } \\
\text { granulaires }\end{array}$} & \multicolumn{2}{|c|}{$\begin{array}{c}\text { en plans inter-granulaires, } \\
\text { plus rarement isolé }\end{array}$} \\
\hline $\begin{array}{l}\text { Taux de } \\
\text { remplissage }(V / L)\end{array}$ & \multicolumn{2}{|c|}{0,1 à 0,7} & \multicolumn{2}{|c|}{------- } & \multicolumn{2}{|c|}{0,05 à 0,1} \\
\hline & \multicolumn{6}{|c|}{ Microthermométrie } \\
\hline $\mathrm{TfCO}_{2}\left({ }^{\circ} \mathrm{C}\right)$ & $-58,5 /-56,5(-57)$ & $-68 /-60(-64,3)$ & $-56,6$ & $-66,5 /-61,5(-63,7)$ & ---- & ---- \\
\hline $\mathrm{ThCO}_{2}\left({ }^{\circ} \mathrm{C}\right)$ & non mesurée & $-2 / 25(16)$ & $-4,6$ & $-23 /-16(-22)$ & $\begin{array}{l}--- \\
-\end{array}$ & ---- \\
\hline Tf Clathrate $\left({ }^{\circ} \mathrm{C}\right)$ & $6,5 / 9(8)$ & $6,5 / 9(8,5)$ & $\begin{array}{ll}--- \\
\end{array}$ & $\begin{array}{ll}--- \\
\end{array}$ & $\begin{array}{ll}---- \\
\end{array}$ & ---- \\
\hline $\operatorname{Tm} 1\left({ }^{\circ} \mathrm{C}\right)$ & $-55 /-52(-54)$ & $-57,5 /-53,5(-54)$ & $\begin{array}{ll}--- \\
\end{array}$ & $\begin{array}{c}--- \\
--\end{array}$ & $\approx-30$ & $\approx-30$ \\
\hline $\operatorname{Tm} 2\left({ }^{\circ} \mathrm{C}\right)$ & $-5,5 /-2,5(-4,5)$ & $-9,5 /-1(-5)$ & $\begin{array}{c}-\cdots- \\
-\cdots-\end{array}$ & $\begin{array}{c}--- \\
-\cdots\end{array}$ & $-2,5 / 0(-1)$ & $-1 / 0(-0,5)$ \\
\hline Th totale $\left({ }^{\circ} \mathrm{C}\right)$ & $175 / 325(210)$ & $260 / 370(320)$ & $\begin{array}{ll}--- \\
\end{array}$ & $\begin{array}{ll}--- \\
--\end{array}$ & $90 / 150(105)$ & $90 / 125(110)$ \\
\hline $\begin{array}{l}\text { Salinité } \\
(\% \text { pds éq. } \mathrm{NaCl})\end{array}$ & $2,6 / 6,4(4,2)$ & 1,3 & $\begin{array}{c}--- \\
-1\end{array}$ & $\begin{array}{c}--- \\
-1\end{array}$ & $0 / 5,3(1,8)$ & $0 / 2,95(1,78)$ \\
\hline Densité $\mathrm{CO}_{2}$ & $0,72 / 0,92(0,82)$ & ---- & $0,95 / 0,95(0,95)$ & $1,05 / 1,06(1,05)$ & ---- & ---- \\
\hline \multirow[t]{2}{*}{ Densité totale } & $0,9 / 0,99(0,96)$ & $3,3 / 13,2(7,4)$ & $0,95 / 0,95(0,95)$ & $0,43 / 0,62(0,52)$ & $0,95 / 0,97(0,96)$ & \\
\hline & \multicolumn{6}{|c|}{ Raman } \\
\hline $\mathrm{XCO}_{2}$ & 1 & $0,34 / 0,55(0,45)$ & ---- & $0,38 / 0,75(0,56)$ & 0 & 0 \\
\hline $\mathrm{XCH}_{4}$ & 0 & $0,45 / 0,66(0,55)$ & $\begin{array}{c}-\cdots- \\
--\end{array}$ & $0,25 / 0,62(0,44)$ & 0 & détecté \\
\hline
\end{tabular}

$\mathrm{TfCO}_{2}=$ température de fusion du $\mathrm{CO}_{2}$

$\mathrm{ThCO}_{2}=$ température d'homogéneisation du $\mathrm{CO}_{2}$

Tf Clathrate $=$ température de fusion du clathrate

Tm1 = température de début de fusion de la glace (température de l'eutectique)

$\mathrm{Tm} 2$ = température de fin de fusion de la glace

Th totale $=$ température d'homogénéisation totale de l'inclusion

\subsubsection{Chronologie des inclusions fluides, relation avec les quartz}

Les relations chronologiques entre les inclusions fluides et leur disposition par rapport à la géométrie des quartz Q1, Q2 et des excroissances sont résumées sur la figure 3. Les inclusions des familles I et II sont, soit dis- persées dans les grains de première génération (Q1), soit en plans intragranulaires limités aux excroissances de ces grains (figure 2f). Dans les grains de quartz Q1 et Q2, les inclusions des familles I et II sont allongées perpendiculairement à l'allongement des grains, et donc perpendiculairement à l'axe $\langle c\rangle$ dans le cas de quartz en 
Figure 3. Schéma synthétique des relations entre les inclusions fluides et les différentes générations de quartz.

Figure 3. Schematic relationships between various types of fluid inclusions and generations of quartz.
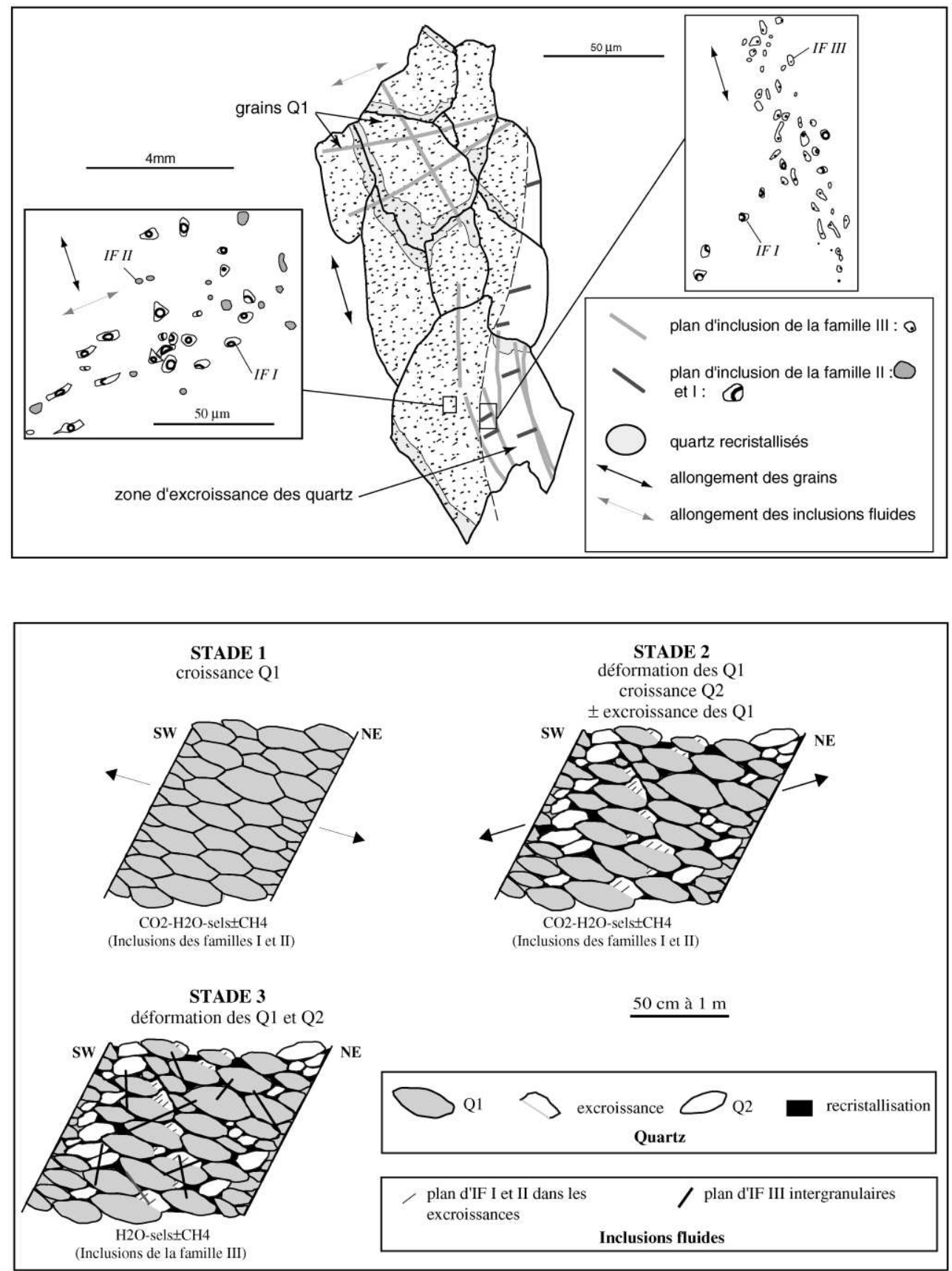

Figure 4. Modèle de formation des veines de quartz aurifère du district de Lo Increible.

Figure 4. Model of formation of gold-bearing quartz veins of the Lo Increible district. peigne (figures $2 d$ et $2 e$ ). Ces inclusions sont alors considérées comme des inclusions primaires, puisqu'elles se parallélisent ainsi aux zones de croissance [7]. Inversement, ces mêmes inclusions, lorsqu'elles sont observées en plans dans les excroissances, sont assimilables à des inclusions pseudo-secondaires d'après les critères classiques [10]. Ces plans correspondent à des microfractures contemporaines de la formation de l'excroissance. De tels plans ont été précédemment décrits dans des grains de quartz en peigne, dans lesquels leur orientation était perpendiculaire à l'allongement de ces quartz [11]. Ainsi, le piégeage des inclusions I et II, qu'elles soient considérées comme primaires (dans les quartz en peignes Q1) ou pseudo-secondaires (dans les excroissances), apparaît contemporain de ces deux types de quartz. Les inclusions fluides de la famille III remplissent des microfractures cicatrisées se recoupant entre elles et traversant les grains de quartz (figure $2 g$ ). Ces fluides sont les plus tardifs.

\section{Modèle de formation des filons aurifères d'El Callao}

Les veines aurifères d'El Callao se sont formées en trois stades (figure 4). Les deux premiers, caractérisés 
par des textures d'ouverture et de remplissage, sont parfois représentés dans un même échantillon (figure 4). Au cours du premier stade, les quartz en peigne (Q1) cristallisent et piègent des fluides à $\mathrm{CO}_{2}-\mathrm{H}_{2} \mathrm{O}-$ sels- $-\left( \pm \mathrm{CH}_{4}\right)$ et à $\mathrm{CO}_{2}\left( \pm \mathrm{CH}_{4}\right)$ (inclusions I et II). Les inclusions sont localisées au cœur des grains, alignées selon des directions parallèles aux zones de croissance. Lors du deuxième stade, une deuxième génération de quartz en peigne (Q2) cristallise en bordure des filons, alors que cette deuxième phase de remplissage se traduit au cœur du filon par la formation d'excroissances sur les grains Q1 (figure 4). Ces derniers subissent alors une première déformation (recristallisation). Des inclusions fluides appartenant aux familles I et II sont également piégées pendant cet épisode. Toutefois, leurs habitus diffèrent et les inclusions I et II se rencontrent le long de plans intragranulaires dans les excroissances (figure 3). Ce modèle implique que les deux stades d'ouverture se succèdent dans un intervalle de temps assez bref, car les inclusions des familles I et II se retrouvent dans les deux types de structures. Dans un dernier stade, le piégeage des inclusions fluides tardives (famille III, $\mathrm{H}_{2} \mathrm{O}-$ sels- $\left.\left( \pm \mathrm{CH}_{4}\right)\right)$ s'effectue le long de microfractures qui recoupent toutes les générations de quartz (stade 3, figure 4). La déformation et la recristallisation des quartz Q1 et Q2 se poursuivent.

\section{Discussion}

Le modèle de formation présenté ci-dessus montre des conditions de piégeage similaires pour les inclusions flui- des des familles I et II, de compositions chimiques différentes. Un tel schéma peut s'expliquer, comme le rappellent Anderson et al. [1], soit : (i) par l'exsolution d'un fluide parental en deux phases immiscibles; (ii) par un mélange de deux fluides homogènes et contemporains (i.e. piégeage hétérogène); ou (iii) par des modifications ultérieures au piégeage, telles que le necking-down ou le leakage [10]. L'observation microscopique n'ayant montré aucune preuve d'étranglement ou de perte du contenu des inclusions fluides, la dernière hypothèse est écartée. Les caractéristiques de l'immiscibilité d'un fluide parental sont: (i) variation des densités, (ii) co-existence d'inclusions riches en $\mathrm{H}_{2} \mathrm{O}$ ou en $\mathrm{CO}_{2}$, qui homogénéisent respectivement en phase vapeur et en phase liquide, dans des températures du même ordre de grandeur [9]. Les fluides ici étudiés ne remplissent pas ces conditions. Les compositions, ainsi que les degrés de remplissage des inclusions I et II décrivent tous les intermédiaires entre un pôle riche en $\mathrm{H}_{2} \mathrm{O}$ et un pôle riche en $\mathrm{CO}_{2}$. Cette dernière caractéristique favorise I'hypothèse d'un piégeage hétérogène de deux fluides, I'un riche en $\mathrm{CO}_{2}$ $\left( \pm \mathrm{CH}_{4}\right)$ à forte densité (famille I), I'autre aqueux (famille II). Cet exemple représente donc un excellent cas d'étude, qui permet de mieux comprendre l'évolution physicochimique des fluides lors de la formation/déformation des minéralisations filoniennes. Il illustre ainsi parfaitement l'intérêt de l'analyse combinée de la texture du remplissage des veines et des inclusions fluides.

Remerciements. Les auteurs remercient E. Dumas de la compagnie Auriferos El Callao C.A., ainsi que M. Faure et C. Ramboz pour les discussions fructueuses sur le terrain et au laboratoire.

\section{Références}

[1] Anderson M.R., Rankin A.H., Spiro B., Fluid mixing in the generation of mesothermal gold mineralisation in the Transvaal Sequence, Transvaal, South Africa, Eur. J. Mineral. 5 (1992) 933-948.

[2] Bellizia G.A., Pimentel de Bellizia N, Munoz M., Geology and tectonics of northern South America. III. Geodynamics, Bulletin de Geologia, Publ. Esp. 9, Caracas, Venezuela, Ministerio de Energia y Minas (1981) 7-88.

[3] Borisenko A.S., Study of the salt composition of solutions in gasliquid inclusions in minerals by the cryometric method, Soviet Geol. Geophys. 18 (1977) 11-19.

[4] Brown P., FLINCOR: a microcomputer program for the reduction and investigation of fluid inclusion data, Amer. Mineral. 74 (1989) 1390-1393.

[5] Dowling K., Morrisson G., Application of quartz textures to the classification of gold deposits using North Queensland examples, Econ.
Geol. Monogr. 6 (1989) 342-355.

[6] Dubessy J., Thiery R., Canals M., Modelling of phase equilibria involving mixed gas clathrates: application to the determination of molar volume of the vapour phase and salinity of the aqueous solution in fluid inclusions, Eur. J. Mineral. 5 (1992) 933-948.

[7] Goldstein R.H., Reynolds T.J., Systematics of fluid inclusions in diagenetic minerals, SEPM Short Course 31, SEPM éd. (1994) 199 p.

[8] Kerrick D., Jacobs G.K., A remodified Redlich-Kwong equation for $\mathrm{H}_{2} \mathrm{O}, \mathrm{CO}_{2}$ and $\mathrm{H}_{2} \mathrm{O}-\mathrm{CO}_{2}$ mixtures at elevated pressures and temperatures, Am. J. Sci. 281 (1981) 735-767.

[9] Ramboz C., Pichavant M., Weisbrod A., Fluid immiscibility in natural processes: use and misuse of fluid inclusion data, Chem. Geol. 37 (1982) 29-48.

[10] Roedder E, Fluid inclusions, Mineralogical Society of America, Rev. Mineralogy 12 (1984) 646 p.

[11] Xu G., Fluid inclusions in crack-seal veins at Dugald River, Mount Isa Inlier: implications for palaeostress states and deformation conditions during orogenesis, J. Struct. Geol. 19 (1997) 1359-1368. 